\title{
Captura de carbono ante el cambio climático
}

\author{
José Antonio Benjamín Ordóñez ${ }^{1}$ \\ Omar Masera ${ }^{1}$
}

\section{RESUMEN}

\begin{abstract}
Las actividades humanas, tales como el uso de combustibles fósiles para la producción de energía y los procesos derivados del cambio en el uso del suelo y silvicultura, estan generando grandes emisiones de gases de efecto invernadero (GEI) como dióxido de carbono $\left(\mathrm{CO}_{2}\right)$, monóxido de carbono $(\mathrm{CO})$, clorofluorocarbonados (CFC's), óxidos de nitrógeno $\left(\mathrm{NO}_{\mathrm{x}}\right)$ y metano $\left(\mathrm{CH}_{4}\right)$, principalmente, siendo el $\mathrm{CO}_{2}$ uno de los GEI más importantes por las grandes cantidades en las que se emite. La vegetación tiene la capacidad de asimilar el carbono e incorporarlo a su estructura, es decir, lo fija y lo mantiene almacenado por largos periodos, a través de la fotosíntesis. Es por esta razón que los bosques son importantes sumideros de carbono. El presente trabajo tiene como objetivo describir el proceso de captura de carbono en los ecosistemas forestales y su relación con el cambio climático.
\end{abstract}

PALABRAS CLAVE:

Captura de carbono, cambio climático global, sumideros de carbono, bosques templados.

\begin{abstract}
Human activities have been generating high greenhouse gas emissions (GHG) from two principal sources: fossil fuel used to produce energy and vegetation removal during land use changes and forestry practices. The main $\mathrm{GHG}$ are carbon dioxide $\left(\mathrm{CO}_{2}\right)$, carbon monoxide (CO), clorofluorocarbons (CFC's), nitrogen oxide (NOx) and methane $\left(\mathrm{CH}_{4}\right) . \mathrm{CO}_{2}$ is the most important GHG, since it has the highest emission rates. The vegetation has the capacity to sequester carbon and incorporate it in their structure and keep it for long periods of time, through photosynthesis, therefore forests are important carbon sink. In the present work the carbon sequestration process and its relation with climate change is described.
\end{abstract}

KEY WORDS:

Carbon sequestration, global climate change, carbon sink, template forests. 


\section{INTRODUCCIÓN}

El cambio climático global asociado al aumento potencial de la temperatura superficial del planeta, es uno de los problemas ambientales más severos que se enfrentan en el presente siglo. Este problema se acentúa por el rápido incremento actual en las emisiones de gases de efecto invernadero "GEI" (Bolin et al., 1986) y por las dificultades de reducir en forma sustantiva el incremento de GEl en el futuro próximo (IPCC, 1995). En nuestro país, los principales emisores de GEl son el sector de energía, por el uso de combustibles fósiles, con 83.8 MtC $^{1}$ (Gay y Martínez, 1995), el cambio en el uso del suelo y forestería con 30.2 MtC (Masera et al., 1995a) y los procesos de la industria del cemento con 3.1 MtC, (Gay y Martínez, 1995) dando un total de 117.1 MtC. El Panel Intergubernamental sobre el Cambio Climático (IPCC, 1995) estimó que las emisiones de México contribuyen con el $1.45 \%$ de las emisiones totales de carbono que se dan en nuestro planeta cada año.

Diferentes autores (Schneider 1989; Houghton y Woodwell,1989; Lashof y Ahuja 1994; Dixon et al., 1994; Masera 1995a; Schimel 1995; Ordóñez, 1998 y 1999) afirman que el dióxido de carbono $\left(\mathrm{CO}_{2}\right)$ es uno de los GEI más importantes y que su emisión a la atmósfera por el cambio en el uso del suelo ocupa el segundo lugar a nivel mundial con una fuerte contribución de las zonas tropicales. La deforestación mundial anual se calcula en 17 millones de hectáreas, lo que significa una liberación anual de cerca de $1.8 \mathrm{GtC}^{3}$; lo que representa el $20 \%$ de las emisiones antropogénicas totales (IPCC, 1992 y 1995; Montoya et al., 1995).
Específicamente para México, este fenómeno es de singular importancia pues, por un lado, se encuentra entre los 20 países con mayores emisiones de estos gases y por el otro, se encuentra entre las regiones más vulnerables a los impactos asociados al cambio climático debido a sus condiciones bioclimáticas y socioeconómicas (Villers y Trejo, 1998; Ordóñez, 1998; Gay, 2000; Milenio, 2001).

A nivel nacional, los bosques son actualmente la segunda fuente de emisiones de GEI, contribuyendo aproximadamente con el $30 \%$ del total (Gobierno de México, 1997). Asimismo, los bosques de México pueden ser extremadamente vulnerables al cambio climático (Villers y Trejo, 1998) y representan un almacén de carbono aproximado de 8 GtC (Masera et al., 1997), cantidad equivalente a las emisiones mundiales actuales de $\mathrm{CO}_{2}$. $\mathrm{La}$ capacidad de almacenamiento de carbono $(C)$ en estos bosques se está perdiendo rápidamente por los procesos de deforestación y degradación de los ecosistemas forestales.

Para proponer estrategias viables dirigidas a la mitigación del cambio climático es imprescindible, por un lado, conocer la dinámica del $\mathrm{C}$ en los ecosistemas forestales $y$, por otra, las modificaciones a los flujos de $\mathrm{C}$ derivadas de los patrones de cambio de uso de suelo. Un primer paso indispensable para lograr este objetivo, es contar con la información básica sobre los contenidos de carbono en los diferentes almacenes del ecosistema (Ordóñez, 1998).

Los principales almacenes de $\mathrm{C}$ en los ecosistemas forestales son el suelo, la

\footnotetext{
Una MtC es igual a un millón de toneladas de carbono

Una tonelada de $\mathrm{CO}_{2}$ equivale a 0.27 toneladas de carbono

Una GtC es igual a mil millones de toneladas de carbono
} 
vegetación y el mantillo. La vegetación es la encargada de incorporar el C atmosférico al ciclo biológico por medio de la fotosíntesis. Los bosques del mundo (templados y tropicales) capturan y conservan más carbono que cualquier otro ecosistema terrestre y participan con el $90 \%$ del flujo anual de carbono entre la atmósfera y el suelo (Apps et al., 1993; Brown et al., 1993; Dixon et al., 1994). De igual manera, el suelo juega un papel muy importante en el ciclaje y almacén del carbono en estos ecosistemas. Como ejemplos de la influencia global de los procesos del suelo están los productos de la desnitrificación, tales como $\mathrm{N}_{2} \mathrm{O}, \mathrm{N}_{2}$, así como los producidos por la descomposición de la materia orgánica del suelo, como el $\mathrm{CO}_{2}, \mathrm{CH}_{4}$ y otros gases asociados al ciclo del $\mathrm{C}$ (Melillo et al., 1989; Mosier et al., 1991). El suelo tiene una gran capacidad de "secuestrar" C (Johnson, 1992), ya que puede acumularlo por miles de años (Schlesinger, 1990).

Un segundo factor clave para determinar los flujos netos de $\mathrm{C}$ a la atmósfera son los cambios en el uso del suelo (Jenny, 1941), mismos que modifican, muchas veces de manera drástica los contenidos de carbono en los distintos almacenes. Estudiar la influencia de los procesos de cambio de uso del suelo en la dinámica de emisiones de C es crítico en el país, pues la deforestación y la degradación del recurso forestal han sido muy aceleradas en las últimas décadas. La tasa de deforestación, no se conoce con precisión, aunque se estima que oscila entre 370 y 670 mil ha año ${ }^{-1}$ para principios de los 90's tan sólo en los bosques templados y las selvas. Masera et al. (1997), afirman que probablemente el valor más alto sea el correcto. De acuerdo con esta última estimación se obtiene que, para los bosques templados, la tasa de deforestación es de $1 \%$ y para las selvas, de $2 \%$ al año (Masera et al., 1995b). Las causas que más impacto tienen en la deforestación son el cambio en el uso del suelo, tales como la conversión a ganadería y a cultivos agrícolas. Estos factores varían en importancia según el tipo de ecosistema (Ordóñez, 1998).

A pesar de ser actualmente fuentes netas de emisión de GEI, los bosques tienen la posibilidad de mitigar GEI por medio de la captura de carbono en diferentes ecosistemas vegetales conocidos como: sumideros (Masera, 1996; Ordóñez, 1998 y 1999).

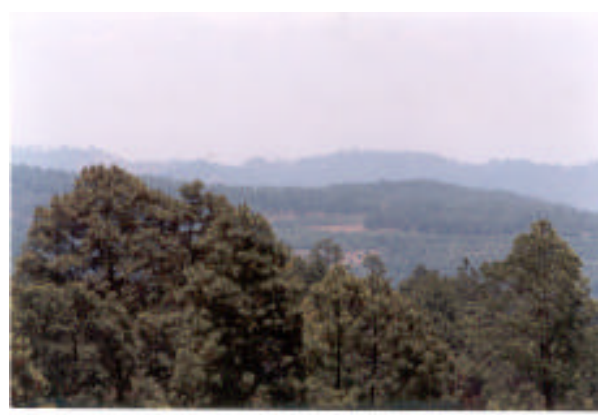

No se cuenta en el país con información detallada sobre los almacenes de $\mathrm{C}$ por tipo de ecosistema y uso del suelo ni de los flujos netos de C derivados de los patrones de cambio de uso del suelo a nivel regional (Hughes et al., 1999; Hughes et al., 2000; Ahedo, 2000). Hasta el momento, los pocos estudios existentes se han concentrado en los ecosistemas tropicales. La información es especialmente deficiente para los bosques templados del Centro y Sur de México, los cuales sufren actualmente un acelerado proceso de deforestación y degradación, con tasas de cambio comparables a las de las selvas del país (Masera, 1996; Ordóñez, 1998 y 1999).

\section{EL CARBONO EN ECOSISTEMAS FORESTALES}

Una vez que el dióxido de carbono $\left(\mathrm{CO}_{2}\right)$ atmosférico es incorporado a los 
procesos metabólicos de las plantas mediante la fotosíntesis, éste participa en la composición de materias primas como la glucosa, para formar todas las estructuras necesarias para que el árbol pueda desarrollarse (e.g. follaje, ramas, raíces y tronco). El árbol al crecer va incrementado su follaje, ramas, flores, frutos, yemas de crecimiento (que en su conjunto conforman la copa); así como altura y grosor del tronco. La copa necesita espacio para recibir energía solar sobre las hojas dando lugar a una competencia entre las copas de los árboles por la energía solar, originando a su vez un dosel cerrado. Los componentes de la copa aportan materia orgánica al suelo, misma que al degradarse se incorpora paulatinamente y da origen al humus estable que, a su vez, aporta nuevamente $\mathrm{CO}_{2}$ al entorno (Ordóñez, 1998 y 1999).

Simultáneamente los troncos, al ir incrementando su diámetro y altura, alcanzan un tamaño tal que puedan ser aprovechados con fines comerciales. De este aprovechamiento se extraen productos como: tablas, tablones y polines, que darán origen a subproductos elaborados como: muebles y casas. Estos productos finales tienen un tiempo de vida determinado después del cual se degradan aportando carbono al suelo y $\mathrm{CO}_{2}$ producto de su descomposición a la atmósfera (Ordóñez, 1998 y 1999).

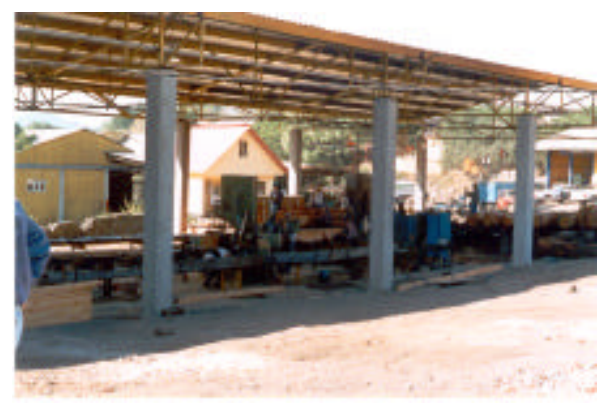

que

Finalmente, durante el tiempo en el carbono se encuentra constituyendo alguna estructura del árbol y hasta que es reemitido (ya sea al suelo o a la atmósfera), se considera que se encuentra almacenado. En el momento de liberación (ya sea por la descomposición de la materia orgánica y/o la quema de la biomasa) el carbono fluye para regresar a su ciclo (Ordóñez, 1998 y 1999).

El diagrama representa los flujos y almacenes de carbono en un ecosistema forestal, donde el follaje, las ramas, las raíces, el tronco, los desechos, los productos y el humus estable son almacenes de carbono, mismos que se reincorporarán al ciclo por descomposición y/o quema de la biomasa forestal.

La estimación precisa de la dinámica de los flujos netos de carbono entre los bosques y la atmósfera (es decir, el balance emisión-captura) es uno de los problemas abiertos más importantes en la discusión sobre cambio climático (IPCC, 1995; Lashof y Ahuja, 1990; Mintzer, 1992; Dixon et al., 1994). Esto es resultado, por un lado, del complejo ciclo biogeoquímico del carbono en los ecosistemas forestales (García y Ordóñez, 1999). En efecto, los procesos de captura-emisión son parte de un sistema con cuatro tipos generales de reservorios de carbono (vegetación, aérea y subterránea, materia en descomposición, suelos, productos forestales), con tiempos de residencia y flujos asociados muy diferentes. Estos reservorios se encuentran estrechamente interrelacionados, lo que hace necesario un enfoque sistémico conocido como: método del sistema total del carbono, (Apps et al., 1993; Dixon et al., 1994) y el uso de modelos de simulación. La problemática es particularmente crítica para los bosques tropicales, en donde no se tiene una buena información de los contenidos de carbono en vegetación y suelo, ni se han podido determinar parámetros para los modelos predictivos existentes (e.g., modelos como: GORKAM, CASFOR, LUCS). 


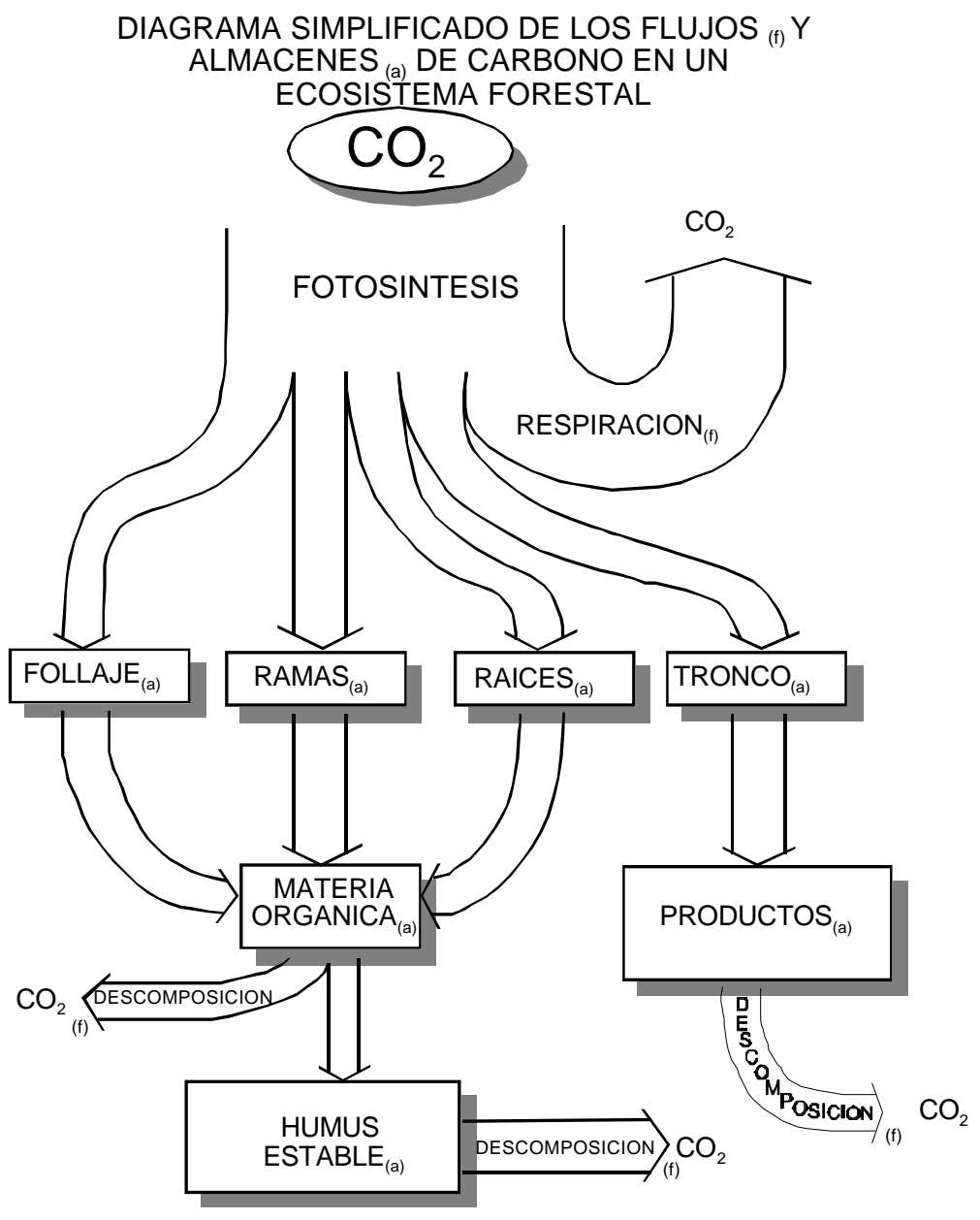

Un segundo problema fundamental para la determinación precisa de las emisiones netas de los ecosistemas forestales, es la gran incertidumbre en los procesos de cambio de uso del suelo, específicamente, cuánto y cómo se pierden los bosques a través del tiempo (IPCC, 1995; Masera et al., 1997). Esto es complicado pues depende de la interacción de factores de tipo biofísico y socioeconómico. Entre los factores físicos y biológicos relacionados con los procesos de cambio de cobertura y uso del suelo destacan las características del relieve del terreno, las propiedades físicas y químicas del suelo, la disponibilidad de fuentes de agua, el estado y la estructura de la vegetación. Además de los procesos naturales de cambio dinámico de los propios ecosistemas como la regeneración y sucesión, hay numerosos factores antropogénicos que promueven la transformación de las áreas naturales, 
impactándolas a distintos niveles y con diferentes intensidades, desencadenando cambios en las relaciones entre la diversidad biológica y el funcionamiento del ecosistema (Naveh y Liebman, 1984; Sauders et al., 1991). No existen muchos trabajos destinados a entender los aspectos finos de la dinámica que ocurre en el paisaje que responde a diversos procesos como la degradación o regeneración de la vegetación. Estos aspectos finos de los procesos de cambio de uso del suelo son, sin embargo, los más importantes desde el punto de vista de la dinámica emisiones-captura de carbono.



\section{LA CAPTURA UNITARIA DE CARBONO}

Para estimar la captura unitaria de carbono se estima el carbono contenido en diferentes almacenes (que pueden ser emitidos o ahorrados; Masera, 1995b; Ordóñez, 1998 y 1999). Estos almacenes incluyen:

\footnotetext{
$C v=$ carbono contenido en la vegetación

$C d=$ carbono contenido en la materia orgánica en descomposición

Cs $=$ carbono contenido en los suelos

$C p=$ carbono contenido en productos forestales (e.g., muebles, papel)

Cf = carbono ahorrado por no utilizar combustibles fósiles (e.g., proyectos bioenergéticos,
}

aclarando que este punto no es un almacen de $\mathrm{C}$ )

Carbono en vegetación (Cv). El carbono en vegetación es la suma del carbono contenido en la biomasa aérea y el carbono contenido en la biomasa de las raíces. La biomasa aérea comprende el tronco, hojas, ramas y follaje; mientras que el carbono contenido en las raíces es definido como la biomasa de las raíces.

Carbono en descomposición (Cd). Es el carbono contenido en la materia orgánica que se encuentra en proceso de descomposición; ésta es originada cuando las estructuras vegetales como hojas, ramas, troncos, son depositadas en el suelo.

Carbono en el suelo (Cs). Es el carbono contenido en las capas que conforman el suelo forestal; este suelo es originado por fragmentación de la roca madre expuesta (material parental), donde se establece un organismo vegetal, que a lo largo del tiempo va formando capas por depositación de materiales, las que al irse acumulando y compactando, almacenan una cierta cantidad de carbono misma que se incrementará por la continuidad del proceso de formación del suelo.

Carbono en productos (Cp). Son los productos forestales almacenes de carbono durante todo el tiempo de vida del producto y cuando este tiempo termina, el carbono se incorporará al ciclo dependiendo del proceso de degradación del producto. Mientras mayor sea la vida media de un producto forestal, el carbono estará almacenado por más tiempo.

Carbono ahorrado por sustitución de combustibles fósiles (Cf). Es una alternativa para contabilizar créditos comercializables por la sustitución de combustibles fósiles (e.g., por el uso de la biomasa forestal para la producción de energía), aclarando que este punto se contempla en el cálculo de la captura 
unitaria pero, no es ni captura, ni secuestro de carbono.

La captura unitaria de carbono $C t$ parte de la siguiente ecuación:

$$
C t=C v+C d+C s+C p+C f
$$

y como resultado de la sumatoria se obtiene $\mathrm{Ct}$ que es igual al carbono total fijado (en ton/ha) o se puede estimar dinámicamente como:

$$
C t=\frac{C v+C d+C s+C p}{T}+\sum C f
$$

donde:

$\mathrm{T}=$ tiempo.

de esta forma, Ct es el promedio del carbono fijado en vegetación, materia en descomposición, suelos y productos forestales, más la suma del carbono ahorrado por la sustitución de combustibles fósiles debido al uso de bioenergía.

En las opciones forestales de mitigación, la captura de carbono se mide en toneladas de carbono por ha (tC/ha/año). Se define asimismo una captura unitaria "neta", es decir la captura que resulta de la diferencia entre llevar a cabo la opción seleccionada y el uso alternativo del uso (por ejemplo, la diferencia entre el contenido de carbono en un bosque y en una parcela agrícola).

La captura unitaria neta de carbono Cnet, por la puesta en práctica de la opción seleccionada está dada como:

$$
\text { Cnet }=\text { Ctp }- \text { Ctref }
$$

donde:

$$
\begin{aligned}
\text { Ctp = } & \text { carbono total fijado en la opción } \\
& \text { de manejo bajo análisis }
\end{aligned}
$$
Ctref $=$ carbono total fijado en el uso (alternativo) del suelo sin proyecto

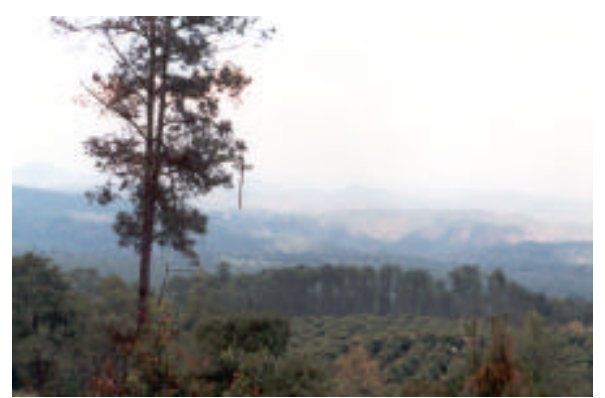

\section{DISCUSIÓN}

Las implicaciones del cambio climático son muchas. Cuando se modifica la temperatura en la Tierra (efecto invernadero), se asocian otros problemas atmosféricos, porque de ello depende la cantidad de evapotranspiración de los océanos, la existencia de nubes, la presencia-ausencia de lluvia y, en consecuencia la producción y la escasez de alimentos. Las regiones semiáridas podrían convertirse en áridas y en los sitios donde llueve mucho podría llover más; es decir hablamos de afectaciones en casi todas las actividades humanas, comenzando con la agricultura (Gay, 2000).

Aunado a esto, tenemos los procesos de deforestación, mismos que liberan grandes cantidades de carbono a la atmósfera y dan origen a problemas de degradación del recurso forestal, de los suelos y como consecuencia de la pérdida de biodiversidad.

Es por estas razones que se están estableciendo mecanismos de desarrollo limpio (MDL), a fin de que los países en desarrollo puedan percibir incentivos por la venta de bonos de captura de carbono 
derivados de la certificación en la reducción de sus emisiones y del establecimiento de proyectos de captura de carbono; sin embargo, aun hay muchas incertidumbres sobre los costos reales de este servicio ambiental y de los requisitos que se pretenden establecer a nivel mundial para este mercado (para mayor información ver: Evaluating carbon offsets from forestry and energy projects: how do they compare?, By Kenneth M. Chomitz, Development Reseach Group, World Bank, March 2000).

\section{CONCLUSIONES}

Dentro de este panorama, una de las tareas prioritarias es llevar a cabo estudios de emisión-captura de carbono a nivel regional en ecosistemas forestales que presenten procesos muy dinámicos de cambio de uso del suelo. Estos estudios deberán permitir, por un lado, estimar más precisamente las densidades de carbono asociadas a las distintas clases de vegetación y, por el otro, desarrollar métodos de cuantificación y predicción sobre el comportamiento de los procesos de emisión captura de carbono bajo diferentes dinámicas de cambio de uso del suelo y de esta forma poder ofrecer opciones de mitigación de gases de efecto invernadero en el corto, mediano y largo plazo, aunado a la posible venta de un servicio ambiental. Es decir, la captura de carbono nos permitirá además, en el largo plazo, contribuir directamente en la mitigación del fenómeno conocido como cambio climático.

\section{RECONOCIMIENTOS}

Se agraceden los comentarios y sugerencias del P. de Biól. Raúl Ahedo, de Ir. Michiel van Breugel y del comité de revisores de la Revista Madera $\boldsymbol{y}$ Bosques, para hacer más claro este documento. Esta investigación ha sido financiada por CONACYT (Proyecto No: 32715-N).

\section{REFERENCIAS}

Ahedo R. 2000. Estimación de biomasa radical y almacenes de carbono en los Tuxtlas Veracruz, México: los cambios con en el uso del suelo. Tesis de Licenciatura, Facultad de Ciencias. UNAM. México, D.F. En Proceso.

Apps, M.J., W.A. Kurz, R.J. Luxmoore, L.O. Nilsson, R.A. Sedjo, R. Schmidt, L.G. Simpson y T.S. Vinson. 1993. Boreal Forests and Tundra. In: Wisniewski, J. y R.N. Sampson (Eds). Terrestrial Biospheric Carbon Fluxes: Quantification and Sources of $\mathrm{CO}_{2}$. Kluwer Academic Publishers. Holanda. p: 39-53.

Bolin, B., B.R. Döös, J. Jager y R.A. Warrick. 1986. The greenhouse effect, climate change and ecosystems. Ed. John Wiley \& Sons.

Brown, S., Ch. Hall, W. Knabe, J. Raich, M. Trexler y P. Woomer. 1993. Tropical forest: their past, present and potential future role in the terrestrial carbon budget. Water, Air and Soil Pollution. No. 70: 71-94.

Dixon, R.K., S. Brown, R.A. Houghton, A.M. Solomon, M.C. Trexler y J. Wisniewski. 1994. Carbon pools and flux of global forest ecosystems. Science 263: 185-190.

García O., F. y A. Ordóñez. 1999. El Papel de los suelos forestales en la captura de carbono. Enviada a Nuestros Bosques.

Gay, C. 2000. El Cambio climático, el problema global más importante del futuro. Gaceta UNAM, Noviembre 13. No. 3411: 12-13. 
Gay, C. y J. Martinez. 1995. Mitigation of emissions of greenhouse gases in Mexico. Interciencia 20(6): 336-342.

Gobierno de México. 1997. Programa Forestal y de Suelo 1995-2000.

Houghton, R.A. y C.M. Woodwell. 1989. Global climate change. Scientific American 260(4):36-40.

Hughes, R.F., J.B. Kauffman y V.J. Jaramillo. 1999. Biomass, carbon, and nutrient dynamics of secondary forests in a humid tropical region of Mexico. Ecology 80(6): 1892-1907.

Hughes, R.F., J.B. Kauffman y V.J. Jaramillo. 2000. Ecosystem-scale impacts of deforestation and land use in a humid tropical region of Mexico. Ecological Applications 10: 515-527.

Intergovermental Panel on Climate Change (IPCC). 1992. Climate Change 1992. The Suplementary Report to the IPCC Scientific Assessment. Cambridge University Press. Cambridge, Inglaterra.

Intergovermental Panel on Climate Change (IPCC). 1995. Climate Change 1995. The Suplementary Report to the IPCC Scientific Assessment. Cambridge University Press. Cambridge, Inglaterra.

Jenny H. 1941. Factors of soil formation. MacGraw-Hill. Nueva York. EUA.

Johnson, D. W. 1992. Effects of forest management on soil carbon storage. In: Natural Sink of $\mathrm{CO}_{2}$. J. Wisniewski y A. E. Lugo (Eds). Kluwer Academic Publs. Palmas de mar, Puerto Rico. p: 83-120.

Lashof, D.A. y D.R. Ahuja. 1990. Relative contributions of greenhouse gas emissions to global warming. Nature 344(5): 529-531.
Masera, O. 1995a. Carbon mitigation scenarios for Mexican forests: methodological considerations and results. Interciencia 20(6): 388-395.

Masera, O. 1995b. Desforestación y degradación forestal en México. Documento de Trabajo 19. Grupo Interdisciplinario de Tecnología Rural Apropiada, A.C. Pátzcuaro, Michoacán, México. 50 p.

Masera, O., T. Hernández, A. Ordóñez y A. Guzmán, 1995a. Land use change and forestry. in preliminary inventory of national greenhouse gases: Mexico. UNEP PROJECT \# GF/4102-92-01 (pp/3011) México, D.F. 56-100. (sept.)

Masera, O., M.R. Bellon y G. Segura. 1995b. Forest management options for sequestering carbon in Mexico. Biomass \& Bioenergy 8(5): 357-367.

Masera, O. 1996. Desforestación y degradación forestal en México. Documento de Trabajo 19. Grupo Interdisciplinario de Tecnología Rural Apropiada, A.C. Pátzcuaro, Michoacán, México. 50 p.

Masera O., M.J. Ordóñez y R. Dirzo. 1997. Carbon emissions from Mexican forests: current situation and long-term scenarios. Climatic Change 35: 265-295.

Milenio. 2001. Crece la amenaza por el cambio climático. Milenio Diario, Lunes 19 de febrero, 2001. p 35.

Melillo, J. M., P.A. Steudler, J.D. Aber y R.D. 1989. In: M.O. Andreae y D.S. Schimel (Eds.) Exchange of trace gases between terrestrial ecosystems and the atmosphere. Wiley \& Sons, p. 263-280.

Mintzer, I.M. 1992. Confronting climate change. Risk implications and 
responses. Cambridge University Press. Cambridge, Inglaterra.

Mosier, A., D. Schimel, D. Valentin, K. Bronson y W. Parton. 1991. Methane and nitrous oxide fluxes in native, fertilized and cultivated grasslands. Nature 350: 330-332.

Montoya, G., L. Soto, B. de Jong, K. Nelson, P. Farias, Pajal Yakac Tic, J. Taylor y R. Tipper. 1995. Desarrollo Forestal Sustentable: Captura de Carbono en las Zonas Tzeltal y Tojolabal del Estado de Chiapas. Instituto Nacional de Ecología, Cuadernos de Trabajo 4. México, D.F.

Naveh, Z. y A. Liebman. 1984. Landscape ecology. Springer-Verlang. Nueva York. p: 3-25.

Ordóñez, A. 1998. Estimación de la captura de carbono en un estudio de caso para bosque templado: San Juan Nuevo, Michoacán. Tesis de Licenciatura, Facultad de Ciencias. UNAM. México D.F.
Ordóñez, A. 1999. Estimación de la captura de carbono en un estudio de caso. Instituto Nacional de Ecología. SEMARNAP. México D.F.

Villers R., L. e I. Trejo V. 1997. assessment of the vulnerability of forest ecosystems to climate change in Mexico. Climate Research 9:8793.

Sauders, D., R. Hoobbs y C. Margules. 1991. Biological consequences of ecosystem fragmentation: a review. Conservation Biology 5: 18-32.

Schimel, D.S. 1995. Terrestrial ecosystems and the carbon cycle: Global Change Biology 1: 77-91.

Schlesinger W. H. 1990. Evidences from chronosequence studies for low carbon-storage potential of soils. Nature 348: 232-234.

Schneider, S.H. 1989. The greenhouse effect: science and policy. Science, 243(10), 271-281. $\diamond$

1 Laboratorio de Bioenergía del Instituto de Ecología del D.E.R.N. de la UNAM. Sur No. 43. Col. Minerva. 09810 México D.F. c.e.:bordonez@ate.oikos.unam.mx

Manuscrito recibido el 16 de octubre de 2000 Aceptado el 23 de febrero de 2001.

Este documento se debe citar como:

Ordóñez, J.A.B. y O. Masera. La captura de carbono ante el cambio climático. Madera y Bosques 7(1):3-12. 